\title{
Saddle Node Bifurcation and Voltage Stability Analysis of Sri Lanka
}

\author{
Dinesh Rangana Gurusinghe
}

\begin{abstract}
In electrical power systems, saddle node bifurcation (SNB) is the voltage stability margin at which voltage collapse takes place. It plays an important role in power system planning and operation as economic and environmental pressures force to operate always closer to the limit of stability. Voltage collapse is associated with the reactive power demands not being met because of limitations in the generation and transmission of reactive power. Determination of the SNB point greatly assists planning and operation of power system. Among various voltage stability analysis (VSA) methods, this paper specifies the continuation power flow (CPF) method as it accurately identifies the SNB point and the critical or the weaker bus. In this paper, power system of Sri Lanka is assessed for diverse applications of VSA by concerning about single element outage or (N-1) criterion and the role of voltage compensation devices. Furthermore, the optimum at the SNB point is verified by eigenvalue analysis.
\end{abstract}

Keywords: saddle node bifurcation, voltage stability analysis, continuation power flow, weakest bus, single element outage, eigenvalue analysis

\section{Introduction}

The continuing electricity demands associated with shortages in installed capacities, brought about by economic and environmental pressures, have led to an increasingly complex system that must operate ever nearer to limits of stability. In electrical power systems, saddle node bifurcation (SNB) is the voltage stability limit at which voltage collapse arises. Of course, all events of voltage collapse are not associated with SNB. Some voltage collapse problems may be due to fast dynamic events that have nothing to do with bifurcation, such as large disturbances that push the system outside its stability region, initiating voltage problems [1].

Determination of SNB plays an important role in the planning and operation of power systems. Voltage collapse typically occurs in power systems, which are heavily loaded, faulted and/or lack of reactive power. Actually, voltage collapse frequently engages an entire power system, although it usually has a relatively larger contribution in one particular part of the power system. Voltage collapse is associated with the reactive power demands of loads not being met because of limitations in the generation and/or transmission of reactive power. Limitations are mainly due to the reactive power limit of generators, characteristics of compensation devices, the action of voltage control devices such as transformer on load tap changers (OLTCs) and the reduced reactive power produced by capacitors at low voltages. The major limitations on the transmission of reactive power are the high reactive power loss on heavily loaded lines and line outages. Reactive power demands of loads increases with the increasing of load or changes in load composition [2].

There are various methods for determining of SNB but the Multiple Power Flow (MPF) method (also known as Direct Method) and the Continuation Power Flow (CPF) method are popular as they accurately identifies the SNB point and the critical or weakest bus [3]. In MPF method, usual power flow determination is performed by increasing loading factor until the system reaches its SNB. The accuracy of the approach depends upon the step size of the loading factor. The main drawback is that number of iterations increase and the accuracy of the solution is little when it closes to the SNB point [4]. In contrast, the CPF method is a mathematical path-following technique applied to solve nonlinear power system equations. This method simply tracks the turning point of the power/voltage characteristics (PV curve) and finds the solution around the SNB point.

Eng. D. R. Gurusinghe, C. Eng., MIE(Sri Lanka), B.Sc Eng. (Hons) (Moratuwa), BIT (Colombo), M. Eng (AIT), M. Tech. (OUSL), Research Associate and Ph. D. Student, Department of Electrical and Computer Engineering, University of Manitoba, Canada. 
This makes the CPF method quite attractive in approximations of the SNB point in a power system. The CPF captures this path-following feature by means of a predictor-corrector scheme that adopts locally parameterized continuation techniques to trace the power flow solution paths [5].

This paper is organized as follows. In Section 2, a brief review of concepts related to the paper is stated. An overview of the power system of Sri Lanka including prominent features of generation, transmission and distribution systems is provided in Section 3. The methodology of the paper including mathematical attributes and the flowchart illustration is discussed in Section 4. Section 5 is concerned to analysis of results. The optimum at the SNB point is verified by eigenvalue analysis. Finally, in Section 6, the main contributions and recommendations of this paper are highlighted.

\section{Brief Review of Concepts}

\subsection{Saddle Node Bifurcation (SNB)}

In the mathematical area of bifurcation theory a saddle-node bifurcation or tangential bifurcation is a local bifurcation in which two fixed points (or equilibria) of a dynamical system collide and annihilate each other. The term 'saddle-node bifurcation' is most often used in reference to continuous dynamical systems [6], [7].

\subsection{Single Element Outage}

If one element of power system is out of service or does not function, it is known as a single element outage or (N-1) contingency criterion. The single element can be either a generator unit or a transformer or a transmission line or any other major element of a power system. However, outage of some elements does not significantly influence to system operation whereas others may cause severe disturbances even the system blackout or brownouts. Most of practical power systems are designed in such a way that they can bear (N-1) contingency criterion without affecting normal system operation.

In voltage stability point of view, outage of single element reduces the system loadability and the SNB takes place at low loading factor.
Therefore, it is essential to analyse the SNB with single element outage.

\subsection{Voltage Compensation Devices}

The role of voltage compensation devices is varying reactive power output to maintain voltage at a set point, which may be either its own terminal bus or a remote bus. Typically voltage compensation devices provide reactive power to the system and it helps to increase the system loadability and the SNB point. There are various types of voltage compensation devices in power systems but capacitors are commonly used as they are comparatively cheap.

In this paper, Static Var Compensator (SVC) is considered as a voltage compensation device, which has a capability to produce reactive power as well as absorb reactive power in the case of high voltage due to excess reactive power. There are other types of compensation devices such as synchronous compensators (STATCOM) and thyristor controlled series compensator (TCSC) are used in power system but they are not consider in this paper.

\subsection{Eigenvalue Analysis}

It has been proved that near system maximum loading point the load flow diverges and power flow Jacobian becomes singular. The eigenvalues associated with the Jacobian matrix provides an indication of the SNB as the smallest eigenvalue reaches zero at the voltage collapsing point [8].

\section{$2.5 \quad$ UWPFLOW}

University of Waterloo Power Flow (UWPFLOW) is a research software tool that has been designed to calculate local bifurcations related to system limits or singularities in the system Jacobian. The program also generates a series of output files that allow further analyses, such as tangent vectors, left and right eigenvectors at a singular bifurcation point, Jacobians, power flow solutions at different loading levels and voltage stability indices [9].

\section{Power System of Sri Lanka}

Ceylon Electricity Board (CEB) is a state owned enterprise in Sri Lanka with the responsibility for most of the generation, transmission and distribution of electric power. The power generation system in the island is 
predominantly owned by CEB while the rest is owned by independent power producers (IPPs). CEB has its own $220 \mathrm{kV}$ and $132 \mathrm{kV}$ Transmission networks, which transmit electricity from generation stations to load centres. In addition, CEB is accountable for $85 \%$ of the power distribution in Sri Lanka and the rest is done by Lanka Electricity Company (LECO), which is one of the subsidiary firms of the CEB [10].

\subsection{Generation System}

The existing generating system in the island has $3,141 \mathrm{MW}$ of capacity and $66 \%$ of capacity is owned by CEB, which includes 1,207MW of hydro, $848 \mathrm{MW}$ of thermal and $3 \mathrm{MW}$ of wind generation capacity. Balance generation capacity of 1,083MW, which includes $842 \mathrm{MW}$ of thermal, 194MW of small-hydro, and 47MW of renewable resources (solar, dendro, biomass and wind), is owned by IPPs [10], [11].

The existing CEB generating system is primarily based on hydro. Fifty-nine per cent of the total existing CEB system capacity is installed at sixteen hydro plants. CEB hydro plants are mainly based on Kelani and Mahaweli river basins and they are cascadelly arranged. Walawe River feeds Samanalawewa hydro plant while Kukule hydro plant, which is a run-of river plant, is located on Kukule Ganga, the main tributary of Kalu Ganga. In addition, CEB owns three small hydropower plants, which are mainly dependent on the irrigation water discharges.

Thermal power generation substantially contributes to the total power generating capacity of the island. CEB's own thermal generation capacity is made up of seven thermal power plants including recently commissioned 300MW coal plant (phase I) at Norochcholai.

IPPs were introduced for power generation after 1996 and their contribution is presently substantial to the healthy operation of the system. Total capacity of IPPs is made up of hundred and five power plants including eleven thermal plants, eighty-five small/mini hydro plants and nine renewable resources based plants [11].

The committed generating system of the island consists of hydro as well as thermal projects. The 150MW Upper Kotmale hydro project, which is run-off-river type, is considered as the largest committed hydro plant. This plant is constructed on the Kotmale Oya, a tributary of Mahaweli Ganga and it is expected to be commissioned in near future.

Mahaweli and Kelani river basins have already been saturated with existing and committed developments but some hydro potential remains to be developed such as 35MW Broadlands, 150MW Uma Oya and 27MW Moragolla. In addition, expansion planning studies have identified 49MW hydro plant that can be developed in Gin Ganga, which is located at southern part of the island [10].

When the system expands it is necessary to identify suitable thermal options because hydro generation is gradually reaching saturation. Among the several thermal options coal fired steam plants have the optimum operational cost. Thus, it is the attractive thermal option in the future in spite of the environmental issues.

\subsection{Transmission System}

The existing transmission network covers the entire island except the northern part. The network operates at $220 \mathrm{kV}$ and $132 \mathrm{kV}$ voltage levels. The total route length of $220 \mathrm{kV}$ transmission system is $484 \mathrm{~km}$ and $132 \mathrm{kV}$ system covers $1,752 \mathrm{~km}$. In addition, 55 grid substations ( 5 of $220 / 132 / 33 \mathrm{kV}, 2$ of $220 / 132$ $\mathrm{kV}, 44$ of $132 / 33 \mathrm{kV}$ and 4 of $132 / 11 \mathrm{kV}$ ) are connected to the system [11], [12].

$220 \mathrm{kV}$ transmission system comprises overhead transmission lines while a few underground cable feeders are used in $132 \mathrm{kV}$ system within Colombo city. The majority of grid substations are outdoor substations but a reasonable number of indoor gas insulated switchgear (GIS) substations are also available. In most cases, $11 \mathrm{kV}$ or $33 \mathrm{kV}$ side of the grid substation is constructed with GIS while $132 \mathrm{kV}$ side is outdoor type. But almost all substations located in Colombo city comprises of GIS in both sides due to lack of space.

\subsection{Distribution System}

Voltage levels below $33 \mathrm{kV}$ falls into distribution system and CEB distribution network covers $85 \%$ of the electricity distribution. Rest is done by LECO. The medium voltage (MV) distribution network comprises $24,428 \mathrm{~km}$ of $33 \mathrm{kV}$ primary distribution feeders and $2,256 \mathrm{~km}$ of $11 \mathrm{kV}$ feeders. In addition, there are 122 primary and 21,930 Distribution Substations 
that feed the low voltage (LV) network consisting of $104,153 \mathrm{~km}$ of LV lines in the island. The total number of electricity consumers in Sri Lanka is over 4.5 million.

\subsection{Power System in year 2016}

The power system in year 2016 is considered for this paper due to a couple of reasons. Firstly, the existing power network does not cover the northern peninsula of the island and it is expected to be covered by 2014. Secondly, it is expected to commission a couple of larger coal fired thermal plants as well as a large demand increase especially in northern and eastern part of the island in near future. In addition, it is practically feasible and has enough time to accommodate findings of the study to improve the voltage stability of the system. The system consists of 182 buses including 40 generator buses and 425 transmission elements [12].

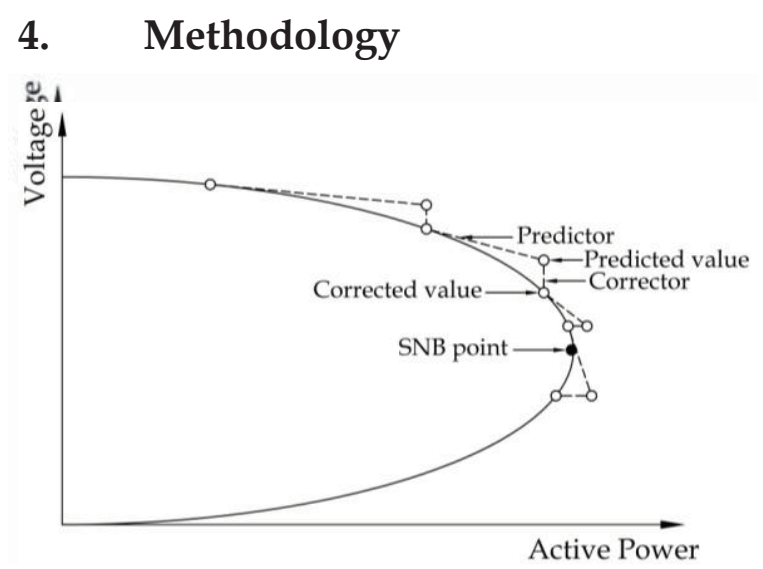

Figure 1 - An illustration of the predictorcorrector method used in the CPF

The Jacobian matrix of power flow equations becomes singular at the SNB point [13], [14]. The CPF method overcomes this problem as it finds successive load flow solutions according to a load scenario. It consists of prediction and correction steps. From a known initial solution, a tangent predictor is used to estimate next solution for a specified pattern of load increase. The corrector step then determines the exact solution using Newton-Raphson technique employed by a conventional power flow analysis. After that a new prediction is made for a specified increase in load, based upon the new tangent vector. Then corrector step is applied. This process goes until the SNB point, where the tangent vector is zero. The illustration of predictor-corrector scheme is depicted in Figure 1.

\subsection{Mathematical Reformation}

Injected active and reactive powers can be written for the $i^{\text {th }}$ bus of an $n$-bus system as;

$$
\begin{aligned}
& P_{i}=P_{G i}-P_{D i}=\sum_{j=1}^{n}\left|V_{i}\right|\left|V_{j}\right|\left|Y_{i j}\right| \cos \left(\theta_{i j}-\delta_{i}+\delta_{j}\right) \\
& Q_{i}=Q_{G i}-Q_{D i}=-\sum_{j=1}^{n}\left|V_{i}\right|\left|V_{j}\right|\left|Y_{i j}\right| \sin \left(\theta_{i j}-\delta_{i}+\delta_{j}\right)
\end{aligned}
$$

where the subscripts $G$ and $D$ denote generation and load demand receptively on the related bus.

$\left|V_{i}\right|,\left|V_{j}\right|=$ voltage magnitude at bus $i$ and bus $j$

$\delta_{i}, \delta_{j}=$ phase angle at bus $i$ and bus $j$

$\left|Y_{i j}\right|, \theta_{i j}=$ magnitude and angle of $(i, j)^{\text {th }}$ element of the bus admittance matrix

When the load level is increased with a load parameter $\lambda$, the system is driven to the SNB. The load is increased as;

$P_{D i}=P_{D 0, i}\left(1+K_{p, i} \cdot \Delta \lambda\right)$

$Q_{D i}=Q_{D 0, i}\left(1+K_{q, i} . \Delta \lambda\right)$

where $P_{D 0, i}, Q_{D 0, i}$ are the initial active and reactive powers while $K_{p, i}, K_{q, i}$ denote active and reactive powers increase direction of load bus $i$.

The active power generation can be modified to;

$P_{G i}=P_{G 0, i}\left(1+K_{g, i} \cdot \Delta \lambda\right)$

where $P_{G 0, i}$ denotes the initial active power generation at bus $i$ while $K_{p, i}$ denote active power increase direction of generator bus $i$.

If $f$ is used to denote the entire set of equations, the power flow model can be expressed as a set of nonlinear algebraic equations represented by;

$$
f(\delta, V, \lambda)=0
$$

where $\delta$ represents voltage phase angles and $V$ denotes voltage magnitudes. The initial solution is obtained for $\lambda=0$. The predictor corrector continuation process can be applied to these equations.

\subsection{Predictor Step}

In the predictor step, a linear approximation is used by taking an appropriately sized step in a 
direction tangent to the solution path. Therefore, the derivative of equation (6) is taken as;

$$
\begin{aligned}
& d\{f(\delta, V, \lambda)\}=f_{\delta} \cdot d \delta+f_{V} \cdot d V+f_{\lambda} \cdot d \lambda=0 \\
& {\left[\begin{array}{lll}
f_{\delta} & f_{V} & f_{\lambda}
\end{array}\right] \cdot\left[\begin{array}{c}
d \delta \\
d V \\
d \lambda
\end{array}\right]=0}
\end{aligned}
$$

In order to solve equation (7), one more equation is needed since an unknown variable $\lambda$ is added to load flow equations. This can be satisfied by setting one of the tangent vector components to +1 or -1 which is also called continuation parameter. Setting one of the tangent vector components +1 or -1 imposes a non-zero value on the tangent vector and makes Jacobian non-singular at the SNB point. As a result equation (7) becomes;

$$
\left[\begin{array}{lll}
f_{\delta} & f_{V} & f_{\lambda} \\
& e_{k} &
\end{array}\right] \cdot\left[\begin{array}{l}
d \delta \\
d V \\
d \lambda
\end{array}\right]=\left[\begin{array}{l}
0 \\
\pm 1
\end{array}\right]
$$

where $e_{k}$ is an appropriately dimensioned row vector with all elements equal to zero except the $k^{\text {th }}$, which is equal to one. At first step $\lambda$ is chosen as the continuation parameter. As the process continues, the state variable with the greatest rate of change is selected as continuation parameter due to nature of parameterization. By solving equation (8), the tangent vector can be found. Then, the prediction can be made as follows;

$$
\left[\begin{array}{l}
\delta \\
V \\
\lambda
\end{array}\right]^{(p+1)}=\left[\begin{array}{l}
\delta \\
V \\
\lambda
\end{array}\right]^{(p)}+\sigma\left[\begin{array}{l}
d \delta \\
d V \\
d \lambda
\end{array}\right]
$$

where the superscript $(p+1)$ denotes the next predicted value. The step size $\sigma$ is chosen so that the predicted solution is within the radius of convergence of the corrector. If it is not satisfied, a smaller step size is chosen.

\subsection{Corrector Step}

In corrector step, the predicted solution is corrected by using local parameterization. The original set of equations is increased by one equation that specifies the value of state variable chosen and it results in;

$$
\left[\begin{array}{c}
f(\delta, V, \lambda) \\
x_{k}-v
\end{array}\right]=[0]
$$

where $x_{k}$ is the state variable chosen as continuation parameter and $v$ is the predicted value of this state variable. Equation (10) can be solved by using a slightly modified NewtonRaphson power flow method (altered only in that one equation and one additional state variable are involved).

\subsection{Parameterization}

Selection of continuation parameter is important in continuation power flow. Continuation parameter is the state variable with the greatest rate of change. Initially, $\lambda$ is selected as continuation parameter since at first steps there are small changes in bus voltages and angles due to light load. When the load increases after a few steps the solution approaches the SNB point and the rate of change of bus voltages and angles increase. Therefore, selection of continuation parameter is checked after each corrector step. The variable with the largest change is chosen as continuation parameter. If the parameter is increasing +1 is used, if it is decreasing -1 is used in the tangent vector in equation (8).

In order to summarize the whole continuation power flow process, a flow chart is presented in Figure 2.

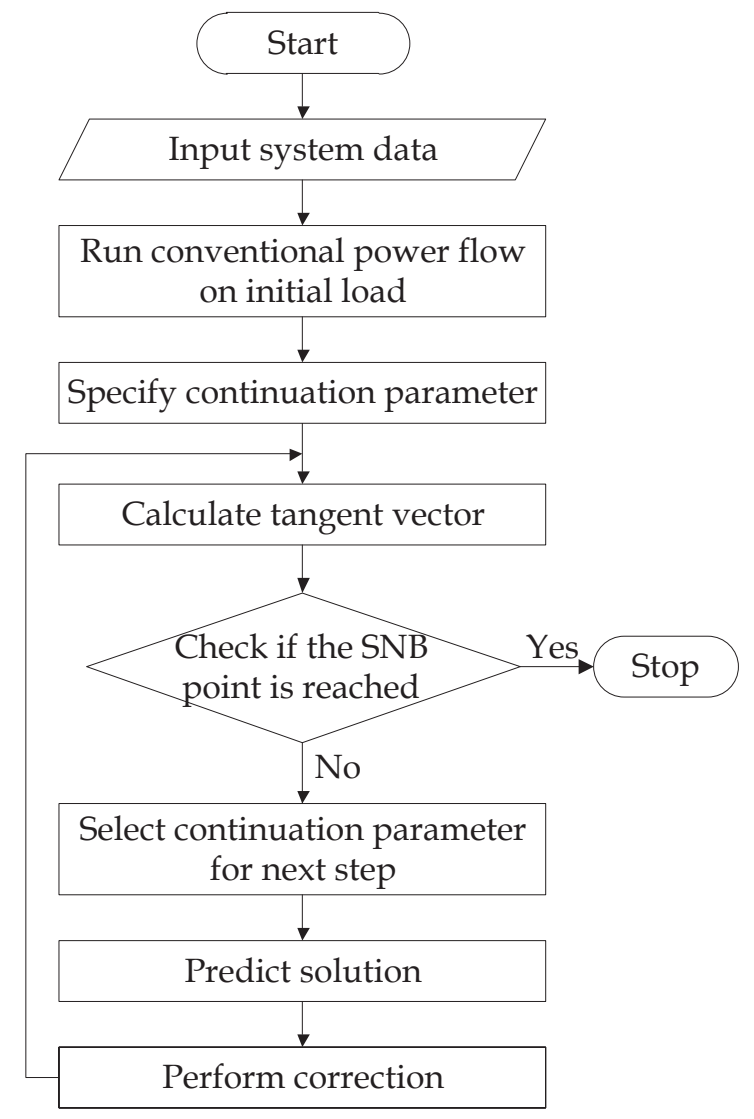

Figure 2 - A flowchart of the CPF [3] 
The CPF is stopped when the SNB point is reached as it is seen in the flowchart. The SNB point is the point where the loading has maximum value. After this point it starts to decrease. The tangent component of $\lambda$ is zero at the critical point and negative beyond this point. Therefore, the sign of $d \lambda$ shows whether the critical point is reached or not.

\subsection{Weakest bus}

The weakest bus means a bus which is nearest to occurring voltage collapse due to lack of reactive power. It means the weakest bus has the maximum ratio of differential change in voltage to differential change in active power demand at the SNB point. In other words, it represents the gradient or the tangent of a PV curve [13], [14]. Therefore, the weakest bus tangent at the SNB point is expressed as;

$$
\left|\frac{d V_{j}}{d P_{j}}\right|=\max \left(\left|\frac{d V_{1}}{d P_{1}}\right|,\left|\frac{d V_{2}}{d P_{2}}\right|, \ldots,\left|\frac{d V_{n}}{d P_{n}}\right|\right)
$$

\section{Results and Discussion}

The SNB point is obtained when loads are increased according to equations (3) and (4), until the voltage collapse has taken place. The loading factor, $\lambda$ at the collapse is taken as the SNB point. The maximum SNB point of a system without violating active and reactive power limits of generators is defined as the optimal operation condition (OOC). This condition can be obtained by changing active power generations according to equation (5) to find better voltage stability.

The solution is obtained from UWPFLOW software and the system optimality is verified with the eigenvalue analysis where the smallest eigenvalue, $\eta_{\min }$ of the power flow Jacobian should be zero at the SNB point.

There are four test cases considered in this paper, expressly, in the determination of the SNB point; a) considering reactive power limits of generators, b) for OOC considering both active and reactive power limits of generators, c) for OOC under (N-1) contingency criterion, and d) for OOC with a voltage compensation device.

\subsection{Considering only reactive power limits}

$220 \mathrm{kV}$ bus at the Victoria power station is considered as the slack bus, which caters excess active power demand due to the load increment and system losses. All other generation stations are maintained their active power at fixed value (constant power) while their reactive power values are allowed to vary within their specified limits. In this case, the critical loading factor (the SNB point), $\lambda_{c}$ is 0.41810 and the smallest eigenvalue is zero. Thus, it verifies the optimality of the solution. Table 1 shows six weak (critical) buses and their voltages at the SNB point. Maho $132 \mathrm{kV}$ bus is the weakest bus followed by Bolawatta $33 \mathrm{kV}$ bus and so on.

Table 1 - Voltages of six weak buses considering only reactive power limits

\begin{tabular}{|l|c|}
\hline \multicolumn{1}{|c|}{ Bus name } & Voltage (kV) \\
\hline Maho 132kV bus & 109.54 \\
\hline Bolawatta 33kV bus & 27.44 \\
\hline Madampe 132kV bus & 111.62 \\
\hline Paddirippu 33kV bus & 28.34 \\
\hline Ampara 33kV bus & 29.15 \\
\hline Puttalum 33kV bus & 27.37 \\
\hline
\end{tabular}

5.2 OOC considering both active and reactive power limits

It is allowed to vary generator active and reactive power within the specified limits so that it shuffles power generation and steers to the highest SNB point of the system, known as the OOC of the system. In this case, $\lambda_{c}$ is 0.66121 and the eigenvalue analysis verified that the solutions represent the SNB as the smallest eigenvalue of the Jacobian equals to zero. Table 2 shows six weak buses and their voltages at the SNB point. $33 \mathrm{kV}$ bus at the Jayawardanapura substation is identified as the weakest bus followed by $11 \mathrm{kV}$ bus at the Dehiwala substation and so on.

Table 2 - Voltages of six weak buses under OOC

\begin{tabular}{|l|c|}
\hline \multicolumn{1}{|c|}{ Bus name } & Voltage (kV) \\
\hline Jayawardanapura 33kV bus & 26.73 \\
\hline Dehiwala 33kV bus & 26.50 \\
\hline Kotugoda 33kV bus (new) & 28.24 \\
\hline Paddirippu 33kV bus & 30.25 \\
\hline Puttalum 33kV bus & 28.22 \\
\hline Ampara 33kV bus & 31.11 \\
\hline
\end{tabular}

5.3 OOC under single element outage or (N-1) contingency criterion

In the case of single element outage or $(\mathrm{N}-1)$ contingency criterion, only significant outages i.e. loss of over $25 \mathrm{MW}$ generations, outage of 
Table 3 - Comparison of $\lambda_{c}$ of 10 critical (N-1) contingency criterions

\begin{tabular}{|l|l|l|c|}
\hline \multicolumn{1}{|c|}{ Outage } & \multirow{2}{*}{$\lambda_{\mathbf{c}}$} & \multicolumn{2}{c|}{ Weakest Bus } \\
\cline { 3 - 4 } & & \multicolumn{1}{c|}{ Name } & Voltage (kV) \\
\hline Kelanitissa : 165MW Generation (220kV bus) & 0.58978 & Bolawatta 33kV bus & 27.26 \\
\hline Galle PS : 80MW Generation & 0.60217 & Panadura 33kV bus & 25.60 \\
\hline Kerawalapitiya : 135MW Generation & 0.60505 & Bolawatta 33kV bus & 27.24 \\
\hline Kelanitissa : 115MW Generation (132kV bus) & 0.60520 & Jayawardanapura 33kV bus & 26.62 \\
\hline Chunnakam : 35MW Generation & 0.61327 & Madampe 33kV bus & 27.90 \\
\hline Norochcholai-New Chilaw 220kV Line & 0.61645 & Paddirippu 33kV bus & 30.33 \\
\hline Kelanitissa : 75MW Generation (33kV bus) & 0.62357 & Paddirippu 33kV bus & 30.54 \\
\hline Trincomalee PS : (150MVA, 220/132kV T/f) & 0.62795 & Bolawatta 33kV bus & 27.27 \\
\hline Samanalawewa : 60MW Generation & 0.63257 & Jayawardanapura 33kV bus & 26.70 \\
\hline Kukule : 35MW Generation & 0.63450 & Kotugoda 33kV bus (new) & 28.19 \\
\hline
\end{tabular}

transmission lines, which carrying over 100MVA and outage of $220 / 132 \mathrm{kV}$ inter-bus transformers are considered. Fifty-three significant single element outages are considered in this study and comparative results of 10 critical outages in terms of voltage stability point of view are given in Table 3 .

It is noted that the loss of $165 \mathrm{MW}$ combined cycle generator connected to the $220 \mathrm{kV}$ bus at Kelanitissa power plant is the most critical outage followed by 80MW diesel generator loss at Galle power station. Eight out of ten critical single element outages are due to generator tripping at major power plants while remaining two are as a result of a critical $220 \mathrm{kV}$ transmission line outage and a 220/132kV inter-bus transformer outage.

In addition to the substations mention in table 3 , it is found that $33 \mathrm{kV}$ buses at Dehiwala, Kolonnawa (new), Ratmalana, Veyangoda, Puttalum and Ampara substations are needed reactive power support under single element outages other than ten critical outages.

\subsection{Installation of 100MVar SVC at Jayawardanapura $33 \mathrm{kV}$ bus}

In the test case where OOC considered both active and reactive power limits of generators, it is identified that Jayawardanapura $33 \mathrm{kV}$ bus is the weakest bus as it requires more reactive power at the SNB point. Therefore, 100MVar SVC is installed to enhance voltage stability of the system. The critical loading factor of the system is significantly increased due to introduction of SVC and the new SNB point is 0.71017 . Table 4 shows six weak buses and their voltages at the SNB point with the SVC. Dehiwala $33 \mathrm{kV}$ bus is the weakest bus followed by Bolawatta $33 \mathrm{kV}$ bus and so on.
Table 4 - Voltages of six weak buses with SVC

\begin{tabular}{|l|c|}
\hline \multicolumn{1}{|c|}{ Bus name } & Voltage (kV) \\
\hline Dehiwala 33kV bus & 26.67 \\
\hline Bolawatta 33kV bus & 27.10 \\
\hline Kotugoda 33kV bus (new) & 28.16 \\
\hline Paddirippu 33kV bus & 29.77 \\
\hline Puttalum 33kV bus & 27.80 \\
\hline Madampe 33kV bus & 27.28 \\
\hline
\end{tabular}

\subsection{Areas for Improvement}

It is important to study the behaviour of OLTC and the optimal tap setting, which furnishes the optimal operating condition of the system. In this paper, SVC is taken as voltage compensation devices, but it is better to check the SNB point with other types of voltage compensators such as shunt capacitors and Static Synchronous Compensators (STATCOM).

\section{Conclusions}

The voltage stability plays an essential role in electric power systems as most of system blackouts or brownouts occur as a result of voltage collapse. Therefore, finding of the voltage collapse point or the SNB point is vital in planning and operation of power system and it assists to avoid possible system collapses especially when the system is heavily loaded. On the other hand, diverse applications of VSA show different SNB points, which are essential for planning and operation.

The CPF method accurately identify the SNB point and furnishes the $\mathrm{OOC}$, where the maximum SNB point takes place while maintaining generator active and reactive power limits. The OOC is imperative in both 
operation and planning point of view. In operation, it indicates how much the existing system can be further loaded while in planning, how many new loads can be added to the existing system.

The optimum is verified by eigenvalue analysis as the smallest eigenvalue at the SNB point is zero.

It is recommended to install sufficient reactive power resources to weakest bus locations identified under normal operation as well as under (N-1) contingency criterion. In this paper, it is found that twelve $33 \mathrm{kV}$ buses at substations, namely Bolawatta, Madampe, Paddirippu, Jayawardanapura, Ampara, Puttalum, Dehiwala, Kotugoda (new), Panadura, Ratmalana, Veyangoda and Kolonnawa (new), require reactive power support to enhance the SNB point of the system.

\section{Acknowledgement}

The author wishes to acknowledge the financial assistance given by the Royal Thai Government (HM Queen Scholarship Program).

\section{References}

1. de Souza, C. Z., Canizares, C. A. \& Quintana, V. H. "New Techniques to Speed up Voltage Collapse Computations using Tangent Vectors," IEEE Trans. Power Systems, Vol. 12, August, 1997, pp. 1380-1387.

2. Alsammak, A. N. B., "Bifurcation and Voltage Collapse in the Electrical Power Systems," Al_Rafidain Engineering, Vol.13, No.1, 2005, pp. 25-41.

3. Ajjarapu, V. \& Christy, C., "The Continuation Power Flow: A New Tool for Steady-State Voltage Stability Analysis," IEEE Trans. Power Systems, Vol. 1, February, 1993, pp. 416-423.

4. Canizares, C. A. \& Alvarado, F. L., "Point of Collapse and Continuation Method for Large AC/DC Systems," IEEE Trans. Power Systems, Vol. 8, February, 1993, pp. 1-8.

5. Ajjarapu, V., Computational Techniques for Voltage Stability Assessment and Control, Springer US, 2007.

6. Kuznetsov, Y. A., "Saddle-node Bifurcation" Department of Mathematics, Utrecht University, Netherlands. IOnlinel. Available:
http://www.scholarpedia.org/article/Saddlenode_bifurcation, Visited, 14 ${ }^{\text {th }}$ November 2011.

7. Mithulananthan, N., Voltage Stability Assessment of Sri Lanka, M. Eng. Dissertation, Energy Field of Study, Asian Institute of Technology, Thailand, 1997.

8. Karbalaei, F., Jadid, S. \& Kalantar, M., “A Novel Method for Fast Computation of Saddle Node Bifurcation in Power Systems using an Optimization Technique," Energy Conversion and Management, Vol. 47, March, 2006, pp. 582-589.

9. Canizares, C. A., Alvarado, F. L. and Zang, S. "UWPFLOW Program" Department of Electrical and Computer Engineering, University of Waterloo, Canada, [Online]. Available: https://ece.uwaterloo.ca/ ccanizar/software/p flow.htm, Visited, 11 ${ }^{\text {th }}$ December 2011.

10. Ceylon Electricity Board, Long Term Generation Expansion Plan 2009-2022, Colombo, Sri Lanka. 2009.

11. Ceylon Electricity Board, Statistical Digest 2011, Colombo, Sri Lanka. 2011.

12. Ceylon Electricity Board, Long Term Transmission Development Plan 2008-2016, Colombo, Sri Lanka, 2009.

13. Kundur, P., Power System Stability and Control, McGraw-Hill, Inc., 1994.

14. Taylor, C.W., Power System Voltage Stability, McGraw-Hill, Inc., 1994. 